\title{
Generalised perturbation equations in bouncing cosmologies
}

\author{
Antonio Cardosd ${ }^{*}$ and David Wands \\ Institute of Cosmology $\& 3$ Gravitation, University of Portsmouth, Portsmouth PO1 2EG, UK
}

(Dated: October 31, 2018)

\begin{abstract}
We consider linear perturbation equations for long-wavelength scalar metric perturbations in generalised gravity, applicable to non-singular cosmological models including a bounce from collapse to expansion in the very early universe. We present the general form for the perturbation equations which follows from requiring that the inhomogeneous universe on large scales obeys the same local equations as the homogeneous Friedmann-Robertson-Walker background cosmology (the separate universes approach). In a pseudo-longitudinal gauge this becomes a homogeneous second-order differential equation for adiabatic perturbations, which reduces to the usual equation for the longitudinal gauge metric perturbation in general relativity with vanishing anisotropic stress. As an application we show that the scale-invariant spectrum of perturbations in the longitudinal gauge generated during an ekpyrotic collapse are not transferred to the growing mode of adiabatic density perturbations in the expanding phase in a simple bounce model.
\end{abstract}

PACS numbers: $04.50 . \mathrm{Kd}, 98.80 . \mathrm{Cq}$

arXiv:0801.1667

\section{INTRODUCTION}

An inflationary expansion in the very early universe can produce the approximately scale-invariant spectrum of adiabatic density perturbations observed in the cosmic microwave background [1], but recently there has been a debate on whether this spectrum could also be generated in cosmological models where the hot big bang phase is preceded by a collapse phase. Among the proposed models are the pre-big bang [2], ekpyrotic [3, 4], and pressureless collapse models [5, 6]. Although many of the models seek to embed the four-dimensional cosmology within a higher dimensional theory, most of the quantitative calculations are done within an effective four-dimensional theory which reduces to general relativity (GR) at sufficiently low energies or late times. However, some modifications to the dynamical equations are required at high energies, close to the bounce, to avoid the singularities present in standard cosmological solutions in GR. This has lead to confusion in the literature, as different modifications appear to give different results for the propagation of perturbations through the bounce. In particular there are conflicting claims as to whether the dominant mode in the longitudinal gauge metric potential $\Psi$, which has an almost scale-invariant spectrum during an ekpyrotic collapse, could in principle give rise to a scale-invariant spectrum in the growing mode of adiabatic density perturbations after the bounce [4, 7, 8, 9, 10, 11].

One approach which has been taken to analyze the transfer of metric fluctuations through the bounce from the collapsing to the expanding phase is to evolve the perturbations through an instantaneous transition along a spacelike hypersurface [12, 13], using the analog of the Israel junction conditions [14], which describe the matching of two solutions of the Einstein equations along a time-like hypersurface. This approach was discussed in Ref. [7], which suggested that generic matching conditions would mix growing and decaying modes across the singular hypersurface, but in Ref. [10] it was argued that no such mixing occurs for adiabatic perturbations. Another approach is to study the evolution of perturbations through a non-singular bounce. This can be done by adding higher order terms in the gravitational action [15, 16, 17], but the resulting field equations are complex and model-dependent.

It is tempting to seek a simpler prescription. In a recent work, Alexander et al [1] assumed that the physics responsible for the bounce does not affect the form of the evolution equation for the perturbations, and the only effect of high-energy modifications at the bounce is to modify the background Hubble rate. They choose a specific cosmological background which leads to exactly soluble equations, and in which one finds that the dominant mode of $\Psi$ during an ekpyrotic collapse couples to the growing mode of density perturbations after the bounce. However, we shall show that this approach implicitly introduces a non-adiabatic component in the perturbations proportional to $\Psi$ on large scales.

Instead we derive a generalised equation for $\Psi$ describing the evolution of adiabatic perturbations in the longwavelength limit for a generalised Friedmann-Robertson-Walker (FRW) background, which reduces to the standard

*Electronic address: antonio.cardoso@port.ac.uk

${ }^{\dagger}$ Electronic address: david.wands@port.ac.uk 
equation when the background is general relativity. This equation is derived by requiring that there exists a longwavelength limit in which the evolution of the perturbed universe is the same as that of the FRW background. That is, we assume that the same physics applies to long-wavelength perturbations as applies to the homogeneous background cosmology [18, 19, 20]. In order to obtain a closed second-order evolution equation for a single metric perturbation we use the gauge freedom to work in a pseudo-longitudinal gauge (defined in an Appendix) without using the Einstein field equations. Adopting the same ansatz for the background Hubble rate as in [11] we find that the dominant mode of $\Psi$ during an ekpyrotic collapse remains decoupled from the growing mode of density perturbations after the bounce.

Finally, we will comment on the relation of our work to previous discussions of generalised perturbation equations on large scales [19, 20, 21]. In particular we identify a generalization of the curvature perturbation on uniform density hypersurfaces, $\zeta$, and show that this quantity is constant in the long-wavelength limit for adiabatic perturbations, even if the gravitational field equations are modified and local conservation of energy is violated.

\section{GENERALISED EQUATIONS FOR THE PERTURBATIONS}

We consider a gravitational theory where homogeneous and isotropic spacetimes obey a Friedmann-type constraint equation, determining the expansion rate of comoving worldlines, $\theta$, and an equation for its evolution with respect to the proper time, $\tau$, along these worldlines,

$$
\begin{aligned}
\theta^{2} & =3 f, \\
\frac{d}{d \tau} \theta & =-\frac{3}{2} g .
\end{aligned}
$$

For example, in loop quantum cosmology a modified effective Friedmann equation $(1)$ can be derived where $f=f(\rho)$ that leads to a cosmological bounce (e.g. 22]), and in Cardassian models [23] $f(\rho) \propto \rho+C \rho^{n}$ have been investigated. In both these examples local energy conservation along comoving worldlines then fixes the form of $g(\rho, p)$. For simplicity we neglect spatial curvature, which we expect to be negligible in the early universe in any case.

In general relativity we then have $f=8 \pi G \rho$ and $g=8 \pi G(\rho+p)$, where $G$ is Newton's constant, $\rho$ is the energy density and $p$ the isotropic pressure of all types of energy-momentum in the universe. More generally, one can always define an effective energy-momentum tensor such that the Einstein tensor $G_{\mu \nu}=8 \pi G T_{\mu \nu}^{\text {eff }}$. From Eqs. (11) and (2) we can identify an effective density and pressure:

$$
\rho^{\mathrm{eff}} \equiv \frac{f}{8 \pi G}, \quad p^{\mathrm{eff}} \equiv \frac{g-f}{8 \pi G}
$$

Conservation of the Einstein tensor, $\nabla^{\mu} G_{\mu \nu}=0$, then requires conservation of the effective energy-momentum tensor, which implies

$$
\frac{d}{d \tau} \rho^{\mathrm{eff}}=-\theta\left(\rho^{\mathrm{eff}}+p^{\mathrm{eff}}\right)
$$

or equivalently, from Eqs. (1) and (2),

$$
\frac{d}{d \tau} f=-\theta g .
$$

However, in the following we will allow $f$ and $g$ to be arbitrary functions of energy, pressure or other variables. In particular we will not assume energy conservation.

We will assume that the spacetime that evolves into our observable Universe can be described by small perturbations about a spatially homogeneous and isotropic spacetime, as in the standard hot big bang model. In particular we will consider scalar perturbations about a spatially flat Friedmann-Robertson-Walker (FRW) metric. The linearly perturbed line-element is given by [24, 27]

$$
d s^{2}=a^{2}\left[-(1+2 \phi) d \eta^{2}+2 B,{ }_{i} d x^{i} d \eta+\left\{(1-2 \psi) \delta_{i j}+2 E,{ }_{i j}\right\} d x^{i} d x^{j}\right],
$$

where $a(\eta)$ is the background scale factor and a comma denotes a partial derivative with respect to the $3 \mathrm{D}$ spatial coordinates. In general one should also consider vector and tensor metric perturbations, but at linear order these decouple from the scalar modes which describe the primordial density perturbations [25].

There is a unit time-like vector field orthogonal to constant- $\eta$ spatial hypersurfaces [26, 27],

$$
N^{\mu}=\frac{1}{a}\left(1-\phi,-B,^{i}\right),
$$


whose expansion rate is given by

$$
\theta=N^{\mu} ; \mu
$$

where a semicolon denotes a covariant $4 \mathrm{D}$ derivative. In terms of the scalar metric perturbations we have

$$
\theta=3 \frac{a^{\prime}}{a^{2}}(1-\phi)-\frac{3}{a} \psi^{\prime}+\frac{1}{a} \nabla^{2} \sigma,
$$

where a prime denotes a derivative with respect to the conformal time $\eta, \nabla^{2}$ denotes the $3 \mathrm{D}$ spatial Laplacian and $\nabla^{2} \sigma$ is the shear, with

$$
\sigma=E^{\prime}-B
$$

At zeroth-order the shear vanishes and the background expansion rate is $\theta_{0}=3 \mathcal{H} / a$, where $\mathcal{H} \equiv a^{\prime} / a$ is the conformal Hubble parameter.

For the zeroth-order homogeneous (FRW) background the equations (11) and (2) can be written as

$$
\begin{aligned}
\mathcal{H}^{2} & =\frac{a^{2}}{3} f_{0}, \\
\mathcal{H}^{\prime}-\mathcal{H}^{2} & =-\frac{a^{2}}{2} g_{0} .
\end{aligned}
$$

Adopting the separate universes viewpoint [19], the same equations (11) and (2) can be used to describe the local evolution in an inhomogeneous universe if we work in the long-wavelength limit where physical length scales are much larger than the time scale set by the expansion rate, $\lambda \gg \theta^{-1}$. This is also sometimes called the ultra-local approximation [29]. Of course the expansion time $\theta^{-1}$ diverges at any cosmological bounce $(\theta=0)$ in the very early universe, but the bounce itself must have a characteristic time scale associated with very high energies and thus very short time scales.

We then can apply Eqs. (11) and (2) where we take $f=f_{0}(\eta)+\delta f(\eta, \mathbf{x})$ and $g=g_{0}(\eta)+\delta g(\eta$, $\mathbf{x})$ and the local expansion rate is given, to first-order, by Eq. (9). Neglecting all spatial gradients, we can then write the first-order equations in terms of the lapse function $\phi$, its derivative, the curvature perturbation $\psi$ and its first and second derivatives,

$$
\begin{aligned}
3 \mathcal{H}\left(\psi^{\prime}+\mathcal{H} \phi\right) & =-\frac{a^{2}}{2} \delta f, \\
\psi^{\prime \prime}-\mathcal{H} \psi^{\prime}+\mathcal{H} \phi^{\prime}+2\left(\mathcal{H}^{\prime}-\mathcal{H}^{2}\right) \phi & =\frac{a^{2}}{2} \delta g .
\end{aligned}
$$

Note that these equations are independent of two of the scalar metric perturbations, $B$ and $E$ in Eq. (6) which determine the anisotropic shear (10), which vanishes in this long-wavelength limit.

For adiabatic perturbations on large scales different patches of the inhomogeneous universe follow the same trajectory in phase space, and the adiabatic perturbations correspond to a perturbation back or forward with respect to the homogeneous background along this trajectory [19]. In this case the hypersurfaces of uniform- $\theta$ and uniform- $(d \theta / d \tau)$ coincide. To first-order this requires $\delta g / g_{0}^{\prime}=\delta f / f_{0}^{\prime}$.

More generally, we can write any perturbation $\delta g$ as a sum of its adiabatic and non-adiabatic parts,

$$
\delta g=\frac{g_{0}^{\prime}}{f_{0}^{\prime}} \delta f+\delta g_{\mathrm{nad}}
$$

where $\delta g_{\text {nad }}$ is automatically gauge-invariant. Indeed, if we identify $f$ with an effective density and $g-f$ with an effective pressure, then $\delta g_{\text {nad }}=8 \pi G\left[\delta p^{\text {eff }}-\left(p_{0}^{\text {eff }^{\prime}} / \rho_{0}^{\text {eff }}{ }^{\prime}\right) \delta \rho^{\text {eff }}\right]=8 \pi G \delta p_{\text {nad }}^{\text {eff }}$. If we assume $f=f(\rho)$ in Eq. (10) and impose energy conservation, so that $d \rho / d \tau+\theta(\rho+p)=0$ along comoving worldlines, then we would require from Eq. (2) that $g=(d f / d \rho)(\rho+p)$ and then $\delta g_{\text {nad }}=(d f / d \rho) \delta p_{\text {nad }}$.

Using Eqs. (15), (11) and (12), we have from Eqs. (13) and (14) that

$$
\psi^{\prime \prime}+\frac{3 \mathcal{H} \mathcal{H}^{\prime}-\mathcal{H}^{\prime \prime}-\mathcal{H}^{3}}{\mathcal{H}^{\prime}-\mathcal{H}^{2}} \psi^{\prime}+\frac{\mathcal{H} \mathcal{H}^{\prime}-\mathcal{H}^{3}}{\mathcal{H}^{\prime}-\mathcal{H}^{2}} \phi^{\prime}+\frac{2 \mathcal{H}^{\prime 2}-\mathcal{H} \mathcal{H}^{\prime \prime}}{\mathcal{H}^{\prime}-\mathcal{H}^{2}} \phi=\frac{a^{2}}{2} \delta g_{\mathrm{nad}} .
$$

Equation (16) includes the two gauge-dependent metric perturbations $\psi$ and $\phi$. However, we still have a choice of temporal gauge. If we choose a pseudo-longitudinal gauge in which $\psi=\phi \equiv \Psi$ (see Appendix A) we find the following evolution equation:

$$
\Psi^{\prime \prime}+\frac{4 \mathcal{H} \mathcal{H}^{\prime}-\mathcal{H}^{\prime \prime}-2 \mathcal{H}^{3}}{\mathcal{H}^{\prime}-\mathcal{H}^{2}} \Psi^{\prime}+\frac{2 \mathcal{H}^{\prime 2}-\mathcal{H} \mathcal{H}^{\prime \prime}}{\mathcal{H}^{\prime}-\mathcal{H}^{2}} \Psi=\frac{a^{2}}{2} \delta g_{\text {nad }}
$$


For adiabatic perturbations the right-hand-side vanishes and we have a homogeneous second-order evolution equation for $\Psi$. We can solve this equation by quadratures to find the general solution [20]

$$
\Psi=C \frac{\mathcal{H}}{a^{2}}+K \frac{\mathcal{H}}{a^{2}} \int \frac{a^{2}\left(\mathcal{H}^{\prime}-\mathcal{H}^{2}\right)}{\mathcal{H}^{2}} d \eta
$$

where $C$ and $K$ are constants of integration. This also has a simpler form [30]:

$$
\Psi=C \frac{\mathcal{H}}{a^{2}}+K\left[-1+\frac{\mathcal{H}}{a^{2}} \int a^{2} d \eta\right]
$$

Although the differential equation (17) has a singular point when $\mathcal{H}^{\prime}-\mathcal{H}^{2}=0$, the solution (19) is clearly regular through a bounce. We show in Appendix A that $\Psi=C \mathcal{H} / a^{2}$ is a solution on all scales in the pseudo-longitudinal gauge, but the second term is only a solution in the long-wavelength limit.

\section{A. General relativity limit}

For general relativity we know the full equations for linear perturbations about a FRW metric. The background expansion rate obeys the Friedmann constraint and evolution equations, (11) and (12), with $f_{0}=8 \pi G \rho_{0}$ and $g_{0}=$ $8 \pi G\left(\rho_{0}+p_{0}\right)$, while we have the following perturbation equations, including the spatial gradients, 24, 27]:

$$
\begin{aligned}
3 \mathcal{H}\left(\psi^{\prime}+\mathcal{H} \phi\right)-\nabla^{2} \psi-\mathcal{H} \nabla^{2} \sigma & =-4 \pi G a^{2} \delta \rho, \\
\psi^{\prime \prime}+2 \mathcal{H} \psi^{\prime}+\mathcal{H} \phi^{\prime}+\left(2 \mathcal{H}^{\prime}+\mathcal{H}^{2}\right) \phi & =4 \pi G a^{2}\left(\delta p+\frac{2}{3} \nabla^{2} \Pi\right),
\end{aligned}
$$

where $\Pi_{i j}-(1 / 3) \delta_{i j} \nabla^{2} \Pi$ is the anisotropic stress. It is straightforward to see that Eqs. (13) and (14) reduce to Eqs. (20) and (21), if we neglect the spatial gradient terms, in general relativity, where $\delta f=8 \pi G \delta \rho$ and $\delta g=8 \pi G(\delta \rho+\delta p)$.

General relativity also supplies a momentum constraint equation, and an evolution equation for the anisotropic shear potential, $\sigma$. In the longitudinal gauge, where $\sigma=0$, this becomes a constraint equation which requires $\psi=\phi$ in the absence of anisotropic stress, $\Pi=0$, 24]. From Eqs. (20), (21) and (23) we obtain

$$
\Psi^{\prime \prime}+3\left(1+c_{s}^{2}\right) \mathcal{H} \Psi^{\prime}+\left[2 \mathcal{H}^{\prime}+\left(1+3 c_{s}^{2}\right) \mathcal{H}^{2}-c_{s}^{2} \nabla^{2}\right] \Psi=4 \pi G a^{2} \delta p_{\text {nad }},
$$

where here $\Psi$ is the curvature perturbation in the longitudinal gauge, and the non-adiabatic pressure perturbation is

$$
\delta p=c_{s}^{2} \delta \rho+\delta p_{\text {nad }},
$$

where $c_{s}^{2} \equiv p_{0}^{\prime} / \rho_{0}^{\prime}$ is the adiabatic sound speed of the matter.

Using Eqs. (11) and (12) it is easy to show that in general relativity Eq. (17) reduces to Eq. (22), neglecting the spatial gradients, with $\delta g_{\text {nad }}=8 \pi G \delta p_{\text {nad. }}$.

More generally, if we use equations (3) to identify an effective density and pressure on large scales, then one can show that our generalised perturbation equation (17) can be written in a "general relativistic" form

$$
\Psi^{\prime \prime}+3\left(1+c_{s}^{2 \mathrm{eff}}\right) \mathcal{H} \Psi^{\prime}+\left[2 \mathcal{H}^{\prime}+\left(1+3 c_{s}^{2 \mathrm{eff}}\right) \mathcal{H}^{2}\right] \Psi=4 \pi G a^{2} \delta p_{\text {nad }}^{\mathrm{eff}},
$$

where the effective adiabatic sound speed is

$$
c_{s}^{2 \mathrm{eff}}=\frac{p_{0}^{\mathrm{eff} \prime}}{\rho_{0}^{\mathrm{eff} \prime}}=\frac{\mathcal{H} \mathcal{H}^{\prime}+\mathcal{H}^{3}-\mathcal{H}^{\prime \prime}}{3 \mathcal{H}\left(\mathcal{H}^{\prime}-\mathcal{H}^{2}\right)} .
$$

\section{APPLICATION TO A SIMPLE BOUNCING COSMOLOGY}

We will now consider the evolution of perturbations in the simple bounce cosmology proposed by Alexander et al in Ref. [11]. They assumed a barotropic fluid in the universe, with pressure $p=w \rho$, and adiabatic density perturbations. To model the bounce they assumed a specific ansatz for the background evolution, with the conformal Hubble rate given by

$$
\mathcal{H}=\frac{q \eta}{\eta^{2}+\eta_{0}^{2}}
$$


where $q \equiv 2 /(1+3 w)$, for which the scale factor has the solution

$$
a=a_{i}\left(\frac{\eta^{2}+\eta_{0}^{2}}{\eta_{i}^{2}+\eta_{0}^{2}}\right)^{\frac{q}{2}}
$$

where $\eta_{i}$ and $a_{i}$ denote the initial values of the conformal time and scale factor, respectively. At early or late times, $|\eta| \gg \eta_{0}$, we recover the general relativistic background evolution

$$
\mathcal{H}=\frac{q}{\eta}
$$

but for $|\eta| \sim \eta_{0}$ we require some unspecified modification of the gravitational field equations to produce the modified expansion given in Eq. (26).

In the general relativistic limit (at early and late times, $|\eta| \gg \eta_{0}$ ) we can use either the GR perturbation equation (22) or its generalisation, (17), to obtain

$$
\Psi^{\prime \prime}+\frac{6(1+w)}{\eta(1+3 w)} \Psi^{\prime}=0
$$

for adiabatic perturbations in the long-wavelength limit. This has the analytic solution

$$
\Psi=D_{ \pm}+S_{ \pm}( \pm \eta)^{-2 \nu}
$$

where $\nu \equiv(5+3 w) /[2(1+3 w)], D_{ \pm}$and $S_{ \pm}$are constants of integration and the + and - signs correspond to the expanding and contracting phases, respectively.

In the ekpyrotic limit, $w \rightarrow \infty$, we obtain

$$
\Psi=D_{ \pm}+S_{ \pm}( \pm \eta)^{-1}
$$

Normalising to initial vacuum fluctuations in the ekpyrotic model gives a scale-invariant spectrum of fluctuations in the growing mode approaching the bounce, $\Psi \sim S_{-}(-\eta)^{-1}$, while the constant mode, $D_{-}$, has a steep blue spectrum [4]. However, observations constrain the spectrum of perturbations in the adiabatic growing mode in the expanding phase, $D_{+}$. have

If we assume that the perturbations are adiabatic in the long-wavelength limit, that is, $\delta g_{\text {nad }}=0$ in Eq. (17), we

$$
\Psi^{\prime \prime}+\frac{4 \mathcal{H} \mathcal{H}^{\prime}-\mathcal{H}^{\prime \prime}-2 \mathcal{H}^{3}}{\mathcal{H}^{\prime}-\mathcal{H}^{2}} \Psi^{\prime}+\frac{2 \mathcal{H}^{\prime 2}-\mathcal{H} \mathcal{H}^{\prime \prime}}{\mathcal{H}^{\prime}-\mathcal{H}^{2}} \Psi=0 .
$$

In the $w \rightarrow \infty$ limit, substituting in Eq. (26) for $\mathcal{H}(\eta)$, Eq. (32) reduces to

$$
\Psi^{\prime \prime}+\frac{2 \eta^{3}-6 \eta_{0}^{2} \eta}{\eta^{4}-\eta_{0}^{4}} \Psi^{\prime}=0
$$

This has the solution

$$
\Psi=C_{1}+C_{2} \frac{\eta}{\eta^{2}+\eta_{0}^{2}},
$$

where $C_{1}$ and $C_{2}$ are constants. Matching the solution in Eq. (34) to the solution in Eq. (31) at both early and late times, $\eta \rightarrow \pm \infty$, we get

$$
D_{+}=D_{-} \quad \text { and } \quad S_{+}=-S_{-}
$$

therefore we see that there is no mixing between the different modes in the early and late time limits. In particular the scale-invariant spectrum during an ekpyrotic collapse, $S_{-}$, couples only to the decaying mode $S_{+}$in the expanding phase, and the adiabatic growing mode in the expanding phase, $D_{+}$, has a steep blue spectrum.

This contrasts with the results presented in [11], where it was assumed that, although the background evolution is modified, Eq. (26), the perturbations are governed by the general relativistic equation (22). Neglecting spatial gradients and setting $\delta p_{\text {nad }}=0$ they obtain the following solution when $w \rightarrow \infty$

$$
\Psi=B_{1}+B_{2} \arctan \left(\frac{\eta}{\eta_{0}}\right)
$$


where $B_{1}$ and $B_{2}$ are constants. Matching this solution to the solution in Eq. (31), at both early and late times, $\eta \rightarrow \pm \infty$, they obtain

$$
D_{+}=D_{-}+\frac{\pi}{\eta_{0}} S_{-} \quad \text { and } \quad S_{+}=-S_{-} .
$$

In this case the mode $D_{+}$receives a contribution from both modes in the collapsing phase.

Comparing our Eq. (17) with Eq. (22) we see that, by modifying the background evolution but using the unmodified GR perturbation equation, the authors of Ref. [1] implicitly introduced a non-adiabatic perturbation in the evolution Eq. (17),

$$
\delta g_{\text {nad }}=-\frac{2}{a^{2}}\left[3 \mathcal{H}\left(1+c_{s}^{2}\right)+\frac{\mathcal{H}^{\prime \prime}+2 \mathcal{H}^{3}-4 \mathcal{H} \mathcal{H}^{\prime}}{\mathcal{H}^{\prime}-\mathcal{H}^{2}}\right]\left(\Psi^{\prime}+\mathcal{H} \Psi\right) .
$$

Note that Alexander et al [11] show that gradient terms are not important on large scales close to the bounce $\left(k \eta_{0} \ll 1\right)$. However, by using the general relativistic equation they have implicitly introduced a non-adiabatic perturbation, $\delta g_{\text {nad }}$, proportional to the longitudinal gauge metric potential $\Psi$, and this is why the $D_{+}$mode in the expanding phase receives a contribution from $S_{-}$which would not otherwise contribute to the growing mode in the expanding phase.

Mode mixing is certainly possible, indeed it happens in general relativity, but only on finite scales due to $\nabla^{2} \Psi \neq 0$ (see e.g., [31]). But mixing occurs due to spatial gradients and hence is suppressed on large scales. Of course we cannot say that there is no theory in which a non-adiabatic perturbation of the form given in Eq. (38) could occur, but one would need a physical model for the appearance of non-adiabatic source terms unsuppressed on large scales (e.g., a non-adiabatic perturbation of a multi-component system). We have shown that by assuming only adiabatic perturbations on large scales, derived from linear perturbations about the background solutions to Eqs. (1) and (2), no such mixing occurs.

\section{CONCLUSIONS}

In this paper we have derived a generalised equation for the evolution of scalar metric perturbations in the longwavelength limit, for a generalised Friedmann-Robertson-Walker (FRW) background, which reduces to the standard equation for the longitudinal metric perturbation in general relativity.

Our results are consistent with previous work [19, 21] which pointed out that the curvature perturbation on uniformdensity hypersurfaces [19, 28],

$$
\zeta \equiv-\psi-\frac{\mathcal{H}}{\rho_{0}^{\prime}} \delta \rho,
$$

is conserved for adiabatic perturbations on large scales assuming only local conservation of energy (see also [32, 33, 34]). We can define a generalization

$$
\zeta_{f} \equiv-\psi-\frac{\mathcal{H}}{f_{0}^{\prime}} \delta f
$$

which is the gauge-invariant definition of the curvature perturbation, $-\psi$, on uniform-expansion hypersurfaces, where $\delta f=0$. Using Eqs. (11) and (13) we can write

$$
\zeta_{f}=\frac{1}{\mathcal{H}^{\prime}-\mathcal{H}^{2}}\left[\mathcal{H} \psi^{\prime}+\left(\mathcal{H}^{2}-\mathcal{H}^{\prime}\right) \psi+\mathcal{H}^{2} \phi\right] .
$$

In general relativity the uniform-density and uniform-expansion hypersurfaces coincide in the long-wavelength limit and hence $\zeta_{f}=\zeta$. Using Eqs. (13) and (14) for the evolution of perturbations on large scales we obtain

$$
\zeta_{f}^{\prime}=\frac{\mathcal{H}}{\mathcal{H}^{\prime}-\mathcal{H}^{2}} \frac{a^{2}}{2} \delta g_{\mathrm{nad}} .
$$

We see that $\zeta_{f}$ is constant in the large-scale limit for modified gravitational field equations, even allowing for nonconservation of energy, if the perturbations are adiabatic, i.e., $\delta g_{\text {nad }}=0$ in Eq. (15).

We see that the growing mode solution (in an expanding universe) for the pseudo-longitudinal gauge perturbation, $\Psi_{+} \propto K$ in Eq. (18), corresponds to $\zeta_{f}=K$, where $K$ is a constant of integration. The decaying mode $\Psi_{-}=C \mathcal{H} / a^{2}$ 
does not contribute to the curvature perturbation $\zeta_{f}$ and is the residual gauge mode in the definition of the pseudolongitudinal gauge (see Appendix A). The other physical degree of freedom in the pseudo-longitudinal gauge resides in the anisotropic shear potential, $\tilde{\sigma}$, which does not affect $\zeta_{f}$ or the large-scale evolution equation (17) in the longwavelength limit.

The same solution (18) was given by Bertschinger [20] in modified gravity for the longitudinal gauge metric perturbation assuming that the trace-free spatial part of the Einstein tensor vanishes (equivalent to assuming vanishing anisotropic stress in GR). We have shown that it is possible to use the solution (18) for adiabatic cosmological perturbations on large scales in a pseudo-longitudinal gauge without making any assumption about the gravity theory, energy conservation or anisotropic stress. The GR solution for the shear in the pseudo-longitudinal gauge in the absence of anisotropic stress (e.g., in a conventional hot big bang phase) is given by $\tilde{\sigma}=\tilde{C} / a^{2}$, where $\tilde{C}$ is a constant of integration. In this case we can perform a further gauge transformation $\delta \eta_{\ell}=\tilde{\sigma}$ [see Eq. (A3)] to obtain the usual longitudinal gauge curvature perturbation, $\Psi_{\ell}=\Psi+\tilde{C} \mathcal{H} / a^{2}$, which shows that in the GR limit the longitudinal and pseudo-longitudinal solutions differ only in the decaying mode, $\propto \mathcal{H} / a^{2}$.

We applied our formalism to adiabatic perturbations in a simple cosmological bounce model, assuming a specific ansatz for the background evolution introduced in Ref. [11]. We took the limit where the equation of state of the matter present in the universe $w=p / \rho$ goes to infinity, corresponding to an ekyprotic model. We conclude that the dominant mode of the curvature perturbation after the bounce does not receive a contribution from the growing mode in the collapse phase. This result is in contrast with the result presented in Ref. [1], where they have assumed that, although the background evolution is modified, the perturbations are governed by the same equation as in general relativity. We have shown that such an approach is equivalent to introducing a non-adiabatic component proportional to $\Psi$ on large scales.

Since it is not possible to unambiguously separate perturbations from the background in the large scale limit where spatial gradients are negligible, we argue that if the background equations are modified then the perturbation equations must also be modified in the large scale limit. And Eq. (17), not Eq. (22), is the consistent generalisation of the evolution equation for long-wavelength linear perturbations given a modified background solution for $\mathcal{H}(\eta)$.

The general nature of the perturbation equation (17) and solution (19), derived only assuming generalised gravity field equations of the form given in (11) and (2), implies that the growing mode of metric perturbations (31) in an ekpyrotic phase will only contribute to the decaying mode in an expanding phase, independently of the details of the bounce or the expanding phase.

\section{Acknowledgements}

The authors are grateful to Alexei A. Starobinsky for drawing their attention to Ref. [30]. AC is supported by FCT (Portugal) PhD fellowship SFRH/BD/19853/2004. DW is supported by STFC.

\section{APPENDIX A: PSEUDO-LONGITUDINAL GAUGE}

The metric perturbations $\phi$ and $\psi$ given in Eq. (6) and $\sigma$ defined in Eq. (10) are gauge-dependent. Under a change of time coordinate $\eta \rightarrow \eta+\delta \eta$ we have [24, 27]

$$
\begin{aligned}
& \phi \rightarrow \tilde{\phi}=\phi-\mathcal{H} \delta \eta-\delta \eta^{\prime} \\
& \psi \rightarrow \tilde{\psi}=\psi+\mathcal{H} \delta \eta \\
& \sigma \rightarrow \tilde{\sigma}=\sigma-\delta \eta
\end{aligned}
$$

Therefore we can always choose

$$
\delta \eta^{\prime}+2 \mathcal{H} \delta \eta=\phi-\psi
$$

such that from Eqs. (A1) and (A2) we have $\tilde{\psi}=\tilde{\phi} \equiv \Psi$. This fixes the gauge up to an arbitrary constant of integration $C(\mathbf{x})$, which is similar to what occurs in the synchronous gauge. Perturbations in this pseudo-longitudinal gauge are given in terms of metric perturbations in an arbitrary gauge by substituting Eq. (A4) in either Eq. (A1) or (A2), to obtain

$$
\Psi=\psi+\frac{\mathcal{H}}{a^{2}} \int a^{2}(\phi-\psi) d \eta
$$


We see that we can write the general solution for $\Psi$ as $\Psi_{+}(\eta)+\Psi_{-}(\eta)$, where $\Psi_{-}(\eta)=C \mathcal{H} / a^{2}$ decays in an expanding universe for $w>-1$.

In general relativity we have a field equation coming from the trace-free part of the Einstein tensor which requires

$$
\psi-\phi=8 \pi G a^{2} \Pi-\sigma^{\prime}-2 h \sigma
$$

where the scalar part of the anisotropic stress is given by $\nabla_{i} \nabla_{j} \Pi-(1 / 3) \delta_{i j} \nabla^{2} \Pi$. In the longitudinal or Newtonian gauge [24] we have $\sigma_{\ell}=0$ by definition, and hence $\psi_{\ell}=\phi_{\ell}$ in the absence of anisotropic stress. More generally, we can define a pseudo-longitudinal gauge in which $\tilde{\psi}=\tilde{\phi}$ and the shear potential is given by

$$
\tilde{\sigma}^{\prime}+2 h \tilde{\sigma}=8 \pi G a^{2} \Pi
$$

Therefore, in the absence of anisotropic stress, $\Pi=0$, we have $\tilde{\sigma} \propto a^{-2}$.

In generalised gravity we may not have a field equation of the same form as Eq. (A6), so we cannot in general identify the pseudo-longitudinal gauge $\tilde{\psi}=\tilde{\phi}$ with a zero-shear gauge. If we assume that there exists a general relativistic limit in which the pseudo-longitudinal gauge reduces to the usual longitudinal gauge (in the absence of anisotropic stress) then this fixes the otherwise arbitrary constant of integration, $C(\mathbf{x})$, and fixes the gauge throughout.

[1] D. N. Spergel et al. [WMAP Collaboration], Astrophys. J. Suppl. 170 (2007) 377 arXiv:astro-ph/0603449.

[2] M. Gasperini and G. Veneziano, Astropart. Phys. 1 (1993) 317 arXiv:hep-th/9211021; Phys. Rept. 373, 1 (2003) arXiv:hep-th/0207130. J. E. Lidsey, D. Wands and E. J. Copeland, Phys. Rept. 337, 343 (2000) arXiv:hep-th/9909061.

[3] J. Khoury, B. A. Ovrut, P. J. Steinhardt and N. Turok, Phys. Rev. D 64 (2001) 123522 arXiv:hep-th/0103239; arXiv:hep-th/0105212 R. Kallosh, L. Kofman and A. D. Linde, Phys. Rev. D 64, 123523 (2001) arXiv:hep-th/0104073.

[4] D. H. Lyth, Phys. Lett. B 524, 1 (2002) arXiv:hep-ph/0106153; J. Khoury, B. A. Ovrut, P. J. Steinhardt and N. Turok, Phys. Rev. D 66, 046005 (2002) arXiv:hep-th/0109050. R. Brandenberger and F. Finelli, JHEP 0111, 056 (2001) arXiv:hep-th/0109004; J. c. Hwang, Phys. Rev. D 65, 063514 (2002) arXiv:astro-ph/0109045.

[5] D. Wands, Phys. Rev. D 60 (1999) 023507 arXiv:gr-qc/9809062.

[6] F. Finelli and R. Brandenberger, Phys. Rev. D 65, 103522 (2002) arXiv:hep-th/0112249.

[7] R. Durrer and F. Vernizzi, Phys. Rev. D 66, 083503 (2002) arXiv:hep-ph/0203275.

[8] C. Cartier, R. Durrer and E. J. Copeland, Phys. Rev. D 67, 103517 (2003) arXiv:hep-th/0301198.

[9] V. Bozza, JCAP 0602, 009 (2006) arXiv:hep-th/0512066.

[10] E. J. Copeland and D. Wands, JCAP 0706, 014 (2007) arXiv:hep-th/0609183.

[11] S. Alexander, T. Biswas and R. H. Brandenberger, arXiv:0707.4679 [hep-th].

[12] J. c. Hwang and E. T. Vishniac, Astrophys. J. 382 (1991) 363.

[13] N. Deruelle and V. F. Mukhanov, Phys. Rev. D 52 (1995) 5549 arXiv:gr-qc/9503050.

[14] W. Israel, Nuovo Cim. B 44S10 (1966) 1 [Erratum-ibid. B 48 (1967 NUCIA,B44,1.1966) 463].

[15] C. Cartier, J. c. Hwang and E. J. Copeland, Phys. Rev. D 64, 103504 (2001) arXiv:astro-ph/0106197.

[16] S. Tsujikawa, R. Brandenberger and F. Finelli, Phys. Rev. D 66 (2002) 083513 arXiv:hep-th/0207228.

[17] M. Gasperini, M. Giovannini and G. Veneziano, Phys. Lett. B 569, 113 (2003) arXiv:hep-th/0306113.

[18] D. S. Salopek and J. R. Bond, Phys. Rev. D 42, 3936 (1990).

[19] D. Wands, K. A. Malik, D. H. Lyth and A. R. Liddle, Phys. Rev. D 62, 043527 (2000) arXiv:astro-ph/0003278.

[20] E. Bertschinger, Astrophys. J. 648, 797 (2006) arXiv:astro-ph/0604485.

[21] A. A. Starobinsky, S. Tsujikawa and J. Yokoyama, Nucl. Phys. B 610 (2001) 383 arXiv:astro-ph/0107555.

[22] P. Singh, K. Vandersloot and G. V. Vereshchagin, Phys. Rev. D 74 (2006) 043510 arXiv:gr-qc/0606032.

[23] K. Freese and M. Lewis, Phys. Lett. B 540 (2002) 1 arXiv:astro-ph/0201229.

[24] V. F. Mukhanov, H. A. Feldman and R. H. Brandenberger, Phys. Rept. 215, 203 (1992).

[25] J. M. Bardeen, Phys. Rev. D 22, 1882 (1980).

[26] H. Kodama and M. Sasaki, Prog. Theor. Phys. Suppl. 78, 1 (1984).

[27] K. A. Malik, arXiv:astro-ph/0101563

[28] J. M. Bardeen, P. J. Steinhardt and M. S. Turner, Phys. Rev. D 28, 679 (1983).

[29] J. K. Erickson, S. Gratton, P. J. Steinhardt and N. Turok, Phys. Rev. D 75, 123507 (2007) arXiv:hep-th/0607164.

[30] D. Polarski and A. A. Starobinsky, Nucl. Phys. B 385 (1992) 623.

[31] C. Gordon, D. Wands, B. A. Bassett and R. Maartens, Phys. Rev. D 63 (2001) 023506 arXiv:astro-ph/0009131.

[32] D. H. Lyth and D. Wands, Phys. Rev. D 68, 103515 (2003) arXiv:astro-ph/0306498.

[33] D. H. Lyth, K. A. Malik and M. Sasaki, JCAP 0505, 004 (2005) arXiv:astro-ph/0411220].

[34] P. Creminelli, A. Nicolis and M. Zaldarriaga, Phys. Rev. D 71, 063505 (2005) arXiv:hep-th/0411270]. 